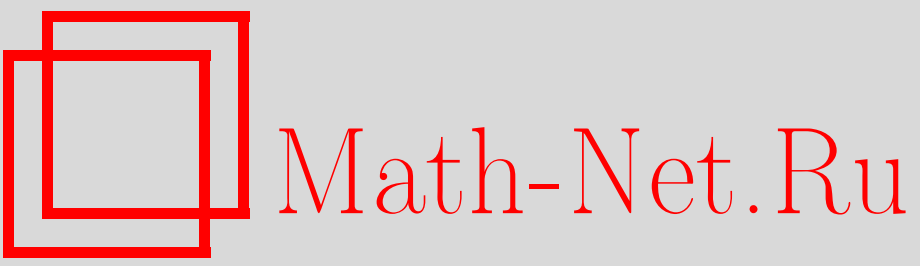

П. А. Яськов, Оценка скорости сходимости в слабом законе больших чисел для процессов эпидемий, Теория вероятн. и ее примен., 2009, том 54, выпуск 3, 533550

DOI: https://doi.org/10.4213/tvp2808

Использование Общероссийского математического портала Math-Net.Ru подразумевает, что вы прочитали и согласны с пользовательским соглашением http://www . mathnet.ru/rus/agreement

Параметры загрузки:

IP : 54.166 .219 .16

26 апреля 2023 г., $10: 13: 11$ 


\title{
ОЦЕНКА СКОРОСТИ СХОДИМОСТИ В СЛАБОМ ЗАКОНЕ БОЛЬШИХ ЧИСЕЛ ДЛЯ ПРОЦЕССОВ ЭПИДЕМИЙ
}

\begin{abstract}
Рассматривается модель General Stochastic Epidemic с немарковским характером развития. В начальный момент население численностью $K$ человек состоит из $[a K]$ зараженных некоторой инфекцией и $K-[a K]$ здоровых, но подозреваемых на возможность заболевания в будущем; здесь $a \in[0,1]$. Предполагается, что изначально инфицированный индивид $i$ выздоравливает через случайное время $\widehat{r}_{i}$, а подозреваемый на инфекцию индивид $j$, заболевающий в (случайный) момент $A_{j}^{K},-$ через случайное время $r_{j}$. Выздоровевший человек приобретает иммунитет и больше не заболевает. В данной работе для эмпирической меры, характеризующей средний путь развития эпидемии, установлена оценка расстояния в идеальной метрике до слабого неслучайного предела (когда $K \rightarrow \infty)$, уточняющая результаты Рейнерт. Полученная оценка явным образом зависит от времени наблюдения за эпидемией и численности населения.
\end{abstract}

Ключевые слова и фразы: процесс эпидемии, слабый закон больших чисел, модель GSE.

1. Введение. Имеются различные стохастические модели, описывающие развитие инфекционных заболеваний (эпидемий), см., например, [1]-[3]. Интерес к этой области исследований обусловлен возможностью не только строить и исследовать нетривиальные модели, но и делать выводы для приложений на практике, см., например, [4], [5].

В данной работе рассматривается обобщение модели General Stochastic Epidemic (GSE), восходящей к [1], которое было предложено в [6]. А именно, пусть имеется население, состоящее из $K$ человек, причем в начальный момент времени $a_{K} K=[a K]$ из них заражено некоторой инфекцией, а остальные $b_{K} K=K-[a K]$ здоровы, но подозреваются на возможность заболевания в будущем; здесь $a \in[0,1],[\cdot]$ целая часть числа. Будем считать, что изначально инфицированный индивид $i\left(i=1, \ldots, a_{K} K\right)$ выздоравливает через случайное время $\widehat{r}_{i}$,

\footnotetext{
* Московский государственный университет им. М.В. Ломоносова, механикоматематический факультет, Ленинские горы 1, 119992 Москва, Россия; e-mail: yaskov_pavel@mail.ru

1) Работа выполнена при поддержке РФФИ (грант № 03-01-00373).
} 
а подозреваемый на инфекцию индивид $j\left(j=1, \ldots, b_{K} K\right)$ заболевает в случайный момент $A_{j}^{K}$, когда некоторый функционал, характеризующий сопротивляемость организма, достигает индивидуального критического уровня $l_{j}$, и процесс его излечения занимает случайное время $r_{j}$. Выздоровевший человек приобретает иммунитет и больше не заболевает. Следуя [7], для описания эпидемии введем следующую конструкцию. Рассмотрим плоскость $\mathbf{R}^{2}$ с декартовой системой координат. По одной оси отложим время заболевания, по другой - время выздоровления. Сопоставим каждому индивиду точку на этой плоскости: изначально инфицированному индивиду $i$ - точку $\left(0, \widehat{r}_{i}\right)$, подозреваемому на заражение индивиду $j$ - точку $\left(A_{j}^{K}, A_{j}^{K}+r_{j}\right)$. Поместим в каждую из введенных точек $\delta$-меру и рассмотрим усреднение этих мер

$$
\xi_{K}:=\frac{1}{K} \sum_{i=1}^{a_{K} K} \delta_{\left(0, \hat{r}_{i}\right)}+\frac{1}{K} \sum_{j=1}^{b_{K} K} \delta_{\left(A_{j}^{K}, A_{j}^{K}+r_{j}\right)} .
$$

Здесь предполагается, что в случае, когда какая-то из введенных точек имеет бесконечную координату, соответствующее ей слагаемое отсутствует в правой части (1.1). Примем $\xi_{K}$ в качестве основной характеристики, описывающей процесс эпидемии. Обозначим $Z_{K}(t)$ среднее число заболевших к моменту времени $t$. Поскольку $Z_{K}(t)$ есть среднее число людей, время заболевания которых наступило до момента $t$, а выздоровление произойдет после момента $t$, то согласно (1.1)

$$
Z_{K}(t)=\xi_{K}([0, t] \times(t,+\infty))=\frac{1}{K} \sum_{i=1}^{a_{K} K} \mathbf{1}_{\left(0, \hat{r}_{i}\right)}(t)+\frac{1}{K} \sum_{j=1}^{b_{K} K} \mathbf{1}_{\left[A_{j}^{K}, A_{j}^{K}+r_{j}\right)}(t) .
$$

Аналогично интерпретируется $\xi_{K}([0, t] \times(s,+\infty))$ - как среднее число людей, заболевших до момента $t$ и не выздоровевших к моменту $s$. Заметим, что на этапе формирования модели вскрывается первая сложность: определяющая характеристика - случайная мера, и для того чтобы эта характеристика стала случайным элементом, надо подходящим образом ввести топологию в пространстве мер на $\left(\mathbf{R}^{2}, \mathscr{B}\left(\mathbf{R}^{2}\right)\right)$.

Наша основная задача состоит в описании асимптотического поведения $\xi_{K}$, а именно в приближении меры $\xi_{K}$ некоторой неслучайной мерой $\mu$ при $K \rightarrow \infty$. В данной работе выводится новая оценка такого приближения, уточняющая результаты [7].

2. Условия на параметры модели. Будем предполагать, что выполнены следующие условия.

$1^{\circ} .\left\{\left(l_{j}, r_{j}\right)\right\}_{j=1}^{b_{K} K}-$ семейство независимых одинаково распределенных (н.о.р.) случайных векторов с неотрицательными компонентами, причем $\Phi-$ функция распределения $r_{j}$, а $\Psi-$ функция распределения $l_{j}$. 
$2^{\circ} \cdot\left\{\widehat{r}_{i}\right\}_{i=1}^{a_{K} K}-$ семейство н.о.р. неотрицательных случайных величин с функцией распределения $\widehat{\Phi}$ и законом распределения $\widehat{\mu}$.

$3^{\circ}$. Семейства $\left\{\left(l_{j}, r_{j}\right)\right\}_{j=1}^{b_{K} K}$ и $\left\{\widehat{r}_{i}\right\}_{i=1}^{a_{K} K}$ независимы.

$4^{\circ} . \widehat{\Phi}(0)=0$ и $\Phi(0)=0$, т.е. зараженный индивид не излечивается мгновенно.

$5^{\circ}$. При каждом $u \in \mathbf{R}_{+}$существует абсолютно непрерывное регулярное условное распределение

$$
\Psi_{u}(t)=\mathbf{P}\left(l_{1} \leqslant t \mid r_{1}=u\right)=\mathbf{1}_{[0,+\infty)}(t) \int_{0}^{t} \psi_{u}(s) d s
$$

и существует $\beta>0$ такое, что для любого $u \geqslant 0$ выполнено неравенство $\left\|\psi_{u}\right\|_{C\left(\mathbf{R}_{+}\right)} \leqslant \beta$.

Обозначим $D_{1}$ множество функций $x: \mathbf{R} \rightarrow[0,1]$, непрерывных справа и имеющих конечные пределы слева. Пусть $\lambda: \mathbf{R}_{+} \times D_{1} \rightarrow \mathbf{R}_{+}-$ некоторый функционал иммунитета. Определим время заражения подозреваемого на заболевание индивида $j$ формулой

$$
A_{j}^{K}:=\inf \left\{t \in \mathbf{R}_{+}: \int_{0}^{t} \lambda\left(s, Z_{K}(\cdot)\right) d s=l_{j}\right\},
$$

где $Z_{K}(t)$ фигурирует в (1.2).

Пусть для любых $t, x(\cdot)$ и $y(\cdot)$ из области определения $\lambda$ выполнены следующие ограничения.

1. Ориентированность на прошлое: $\lambda(t, x(\cdot))=\lambda(t, x(\cdot \wedge t))$.

2. Липшицевость: существует константа $\alpha>0$ такая, что

$$
|\lambda(t, x)-\lambda(t, y)| \leqslant \alpha\|x-y\|_{C[0, t]} .
$$

3. Ограниченность: найдется такое $\gamma>0$, не зависящее от $x$, что $\|\lambda(\cdot, x)\|_{C\left(\mathbf{R}_{+}\right)}<\gamma$.

4. $\lambda(t, x)=0$ тогда и только тогда, когда $x(t)=0$.

Условие 4 гарантирует, что если зараженных людей нет, то эпидемия прекращается. Условие 1 и определение (2.1) позволяют вывести рекуррентную формулу для $A_{j}^{K}$. А именно, если $l_{(k)}$ есть $k$-я порядковая статистика для величин $l_{1}, l_{2}, \ldots, l_{b_{K} K}$, причем $l_{(k)}(\omega)=l_{j_{k}(\omega)}(\omega)$, то для любого фиксированного исхода $\omega$

$$
\begin{aligned}
A_{j_{k}}^{K}=\inf \left\{t \in \mathbf{R}_{+}: \int_{0}^{t} \lambda(\right. & s, \frac{1}{K} \sum_{m=1}^{a_{K} K} \mathbf{1}_{\left(0, \hat{r}_{m}\right)}(\cdot) \\
& \left.\left.+\frac{1}{K} \sum_{n=1}^{k-1} \mathbf{1}_{\left[A_{j_{n}}^{K}, A_{j_{n}}^{K}+r_{j_{n}}\right)}(\cdot)\right) d s=l_{(k)}\right\} .
\end{aligned}
$$

Далее при доказательстве теоремы 4 будет использовано следующее более сильное условие, вытекающее из свойства 2. 
$2^{\prime}$. Существует такое $\alpha>0$, что для любых $(t, x, y) \in \mathbf{R}_{+} \times D_{1}^{2}$

$$
|\lambda(t, x)-\lambda(t, y)| \leqslant \frac{\alpha}{t} \int_{0}^{t}\|x-y\|_{C[0, s]} d s .
$$

3. Пространство случайных мер. Введем ряд обозначений: $M^{b}\left(\mathbf{R}^{2}\right)$ - пространство ограниченных счетно-аддитивных зарядов на $\left(\mathbf{R}^{2}, \mathscr{B}\left(\mathbf{R}^{2}\right)\right) ; \quad M_{1}\left(\mathbf{R}^{2}\right):=\left\{\mu \in M^{b}\left(\mathbf{R}^{2}\right): \inf _{B \in \mathscr{B}\left(\mathbf{R}^{2}\right)} \mu(B) \geqslant 0\right.$, $\left.\sup _{B \in \mathscr{B}\left(\mathbf{R}^{2}\right)}|\mu(B)| \leqslant 1\right\} ; C_{0}\left(\mathbf{R}^{2}\right)$ - пространство непрерывных действительных функций с компактным носителем; $C_{b}(S)$ - пространство непрерывных действительных ограниченных функций на топологическом пространстве $S ; C_{b}^{\infty}\left(\mathbf{R}^{m}\right)$ - пространство бесконечно дифференцируемых функций на $\mathbf{R}^{m}$, все производные которых ограничены.

Рассмотрим на пространстве $M^{b}\left(\mathbf{R}^{2}\right)$ слабую топологию, заданную $C_{0}\left(\mathbf{R}^{2}\right)$, т.е. топологию, база которой состоит из открытых множеств $B_{x, f}=\left\{\nu:\langle\nu, f\rangle\langle x\}\right.$, где $\langle\nu, f\rangle=\int_{\mathbf{R}^{2}} f d \nu, x \in \mathbf{R}$ и $f \in C_{0}\left(\mathbf{R}^{2}\right)$. Согласно [8], $M_{1}\left(\mathbf{R}^{2}\right)$ - польское пространство по отношению к слабой топологии со счетной базой, являющееся слабым компактом. Таким образом, случайные меры $\xi_{K}$ из (1.1) являются случайными элементами со значениями в пространстве $M_{1}\left(\mathbf{R}^{2}\right)$, снабженном слабой топологией и соответствуюшей ей борелевской $\sigma$-алгеброй. Поэтому для них корректно определено вероятностное распределение, и в этом случае можно рассматривать слабую сходимость распределений случайных мер. Введем следующий класс гладких функций:

$$
\begin{aligned}
\mathscr{F}= & \mathscr{F}\left(M_{1}\left(\mathbf{R}^{2}\right)\right) \\
:=\{ & F \in C_{b}\left(M_{1}\left(\mathbf{R}^{2}\right)\right) \mid \exists m \in \mathbf{N} \exists f \in C_{b}^{\infty}\left(\mathbf{R}^{m}\right) \exists \phi_{i} \in C_{b}^{\infty}\left(\mathbf{R}^{2}\right): \\
& F(\cdot)=f\left(\left\langle\cdot, \phi_{i}\right\rangle, i=1, \ldots, m\right),\left\|f^{\prime}\right\| \leqslant 1,\left\|\phi_{i}\right\| \leqslant 1,\left\|\phi_{i}^{\prime}\right\| \leqslant 1, \\
& i=1, \ldots, m\},
\end{aligned}
$$

здесь $\left\|f^{\prime}\right\|=\sum_{i=1}^{m}\left\|f_{x_{i}}^{\prime}\right\|_{C\left(\mathbf{R}^{m}\right)},\left\|\phi^{\prime}\right\|=\sum_{j=1}^{2}\left\|\phi_{x_{j}}^{\prime}\right\|_{C\left(\mathbf{R}^{2}\right)}$. Приведем результат, показывающий, что данный класс является определяющим для слабой сходимости распределений случайных мер в $M_{1}\left(\mathbf{R}^{2}\right)$.

Теорема 1 [8]. Пусть $\xi_{1}, \xi_{2}, \ldots$ - случайньее мерьв в $M_{1}\left(\mathbf{R}^{2}\right)$. Тогда следуюшие условия эквивалентны:

1) $\left\langle\operatorname{Law}\left(\xi_{n}\right), F\right\rangle \rightarrow\langle\operatorname{Law}(\xi), F\rangle$ для любой $F \in \mathscr{F}$ nри $n \rightarrow \infty$;

2) Law $\left(\xi_{n}\right) \stackrel{w}{\Longrightarrow}$ Law $(\xi)$ при $n \rightarrow \infty$, где $\stackrel{w}{\Longrightarrow}$ обозначает слабую сходимость мер.

4. Вспомогательные сведения. Определим следующие операторы, зависящие и от элементарного исхода $\omega \in \Omega$ (далее $D(\mathbf{R})-$ пространство Скорохода, $C(\mathbf{R})$ - пространство непрерывных функций): 
I. $\mathscr{Z}_{K}: D(\mathbf{R}) \rightarrow D_{1}$ и

$$
\mathscr{Z}_{K} f(t):=\frac{1}{K} \sum_{i=1}^{a_{K} K} \mathbf{1}_{\left\{\hat{r}_{i}>t\right\}}+\frac{1}{K} \sum_{j=1}^{b_{K} K} \mathbf{1}_{\left\{f\left(t-r_{j}\right)<l_{j} \leqslant f(t)\right\}} ;
$$

$\mathrm{I}^{\circ} . L_{K}: D(\mathbf{R}) \rightarrow C(\mathbf{R})$ и $L_{K} f(t):=\mathbf{1}_{[0,+\infty)}(t) \int_{0}^{t} \lambda\left(s, \mathscr{Z}_{K} f(\cdot)\right) d s ;$

II. $\mathscr{Z}: D(\mathbf{R}) \rightarrow D_{1}$ и $\mathscr{Z} f(t):=a \mathbf{P}\left(\widehat{r}_{1}>t\right)+b \mathbf{P}\left(f\left(t-r_{1}\right)<l_{1} \leqslant f(t)\right)$, где $b=1-a$;

$\mathrm{II}^{\circ} . L: D(\mathbf{R}) \rightarrow C(\mathbf{R})$ и $L f(t):=\mathbf{1}_{[0,+\infty)}(t) \int_{0}^{t} \lambda(s, \mathscr{Z} f(\cdot)) d s$.

Пусть $F_{K}(t):=\mathbf{1}_{[0,+\infty)}(t) \int_{0}^{t} \lambda\left(s, Z_{K}(\cdot)\right) d s$. Для неубывающей функции $f$ положим $f^{-1}(s):=\inf \{t \geqslant 0: f(t)=s\}$, причем $f^{-1}(s)=+\infty$, если $\|f\|_{C\left(\mathbf{R}_{+}\right)}<s$. Далее, учитывая, что $l_{j}>0$ п.н., из (2.1) для любых $t \geqslant 0$ с вероятностью единица имеем

$$
\begin{aligned}
A_{j}^{K}=F_{K}^{-1}\left(l_{j}\right) & \Rightarrow Z_{K}(t)=\frac{1}{K} \sum_{i=1}^{a_{K} K} \mathbf{1}_{\left\{\hat{r}_{i}>t\right\}}+\frac{1}{K} \sum_{j=1}^{b_{K} K} \mathbf{1}_{\left\{F_{K}^{-1}\left(l_{j}\right) \leqslant t<F_{K}^{-1}\left(l_{j}\right)+r_{j}\right\}} \\
& \Rightarrow Z_{K}(t)=\frac{1}{K} \sum_{i=1}^{a_{K} K} \mathbf{1}_{\left\{\hat{r}_{i}>t\right\}}+\frac{1}{K} \sum_{j=1}^{b_{K} K} \mathbf{1}_{\left\{F_{K}\left(t-r_{j}\right)<l_{j} \leqslant F_{K}(t)\right\}} \\
& \Rightarrow Z_{K}=\mathscr{Z}_{K} F_{K} \Rightarrow L_{K} F_{K}=F_{K},
\end{aligned}
$$

т.е. $F_{K}$ - неподвижная точка $L_{K}$. Из закона больших чисел следует, что для любой неслучайной функции $f \in D(\mathbf{R})$ имеет место сходимость $\mathscr{Z}_{K} f(t) \stackrel{\mathbf{P}}{\rightarrow} \mathscr{Z} f(t)$ при $K \rightarrow \infty$. Поэтому, если бы оператор $L$ имел единственную неподвижную точку $G$, т.е. $L G=G$, то естественно было бы предположить, что для достаточно больших $K$ в некотором смысле $F_{K} \approx G$. В силу (1.1)

$$
\xi_{K}([0, s] \times[0, t])=\frac{1}{K} \sum_{i=1}^{a_{K} K} \mathbf{1}_{\left\{\hat{r}_{i} \leqslant t\right\}}+\frac{1}{K} \sum_{j=1}^{b_{K} K} \mathbf{1}_{\left\{F_{K}^{-1}\left(l_{j}\right) \leqslant s, F_{K}^{-1}\left(l_{j}\right)+r_{j} \leqslant t\right\}},
$$

или

$$
\xi_{K}([0, s] \times[0, t])=\frac{1}{K} \sum_{i=1}^{a_{K} K} \mathbf{1}_{\left\{\hat{r}_{i} \leqslant t\right\}}+\frac{1}{K} \sum_{j=1}^{b_{K} K} \mathbf{1}_{\left\{l_{j} \leqslant F_{K}(s), l_{j} \leqslant F_{K}\left(t-r_{j}\right)\right\}} .
$$

Это приводит к соображению, что в некотором смысле $\xi_{K} \approx \mu$, где неслучайная мера $\mu \in M_{1}\left(\mathbf{R}^{2}\right)$ определяется формулой

$$
\mu([0, s] \times[0, t]):=a \mathbf{P}\left(\widehat{r}_{1} \leqslant t\right)+b \mathbf{P}\left(l_{1} \leqslant G(s), l_{1} \leqslant G\left(t-r_{1}\right)\right),
$$

в которой $G(t)$ полагается равным нулю при $t \leqslant 0$. Формализуем сказанное.

Предположим, что эпидемия наблюдается в течение конечного промежутка времени $[0, T]$. Ограничим меры со всей плоскости $\mathbf{R}^{2}$ на 
квадрат $[0, T]^{2}$, положив по определению $\nu^{T}(\cdot):=\nu\left(\cdot \cap[0, T]^{2}\right)$, где $\nu \in M_{1}\left(\mathbf{R}^{2}\right)$ (очевидно, что при этом $\nu^{T} \in M_{1}\left(\mathbf{R}^{2}\right)$ ). Нам потребуется также следующая теорема, доказательство которой базируется на принципе сжимающих отображений.

Теорема 2 [6]. Для любого $T \geqslant 0$ в пространстве $C[0, T]$ существует и притом единственное решение $G_{T}$ функиионального уравнения $f(t)=\int_{0}^{t} \lambda(s, \mathscr{Z} f(\cdot)) d s$.

3 а м е ч а н и е 1 . Следствием теоремы 2 является существование решения $G$ данного уравнения на $\mathbf{R}_{+}$, причем $G \equiv G_{T}$ на $[0, T]$ в силу единственности $G_{T}$. Поскольку $\lambda \geqslant 0$, то $G$ не убывает, и если $G^{\prime}\left(s_{0}\right)=$ $\lambda\left(s_{0}, \mathscr{Z} G(\cdot)\right)=0$, то по условию 4 , которому отвечает функционал $\lambda$, выполняется равенство $\mathscr{Z} G\left(s_{0}\right)=0$. Но тогда функция $\widehat{G}$, заданная равенствами $\widehat{G}(s)=G(s)$ при $s \in\left[0, s_{0}\right]$ и $\widehat{G}(s)=G\left(s_{0}\right)$ при $s \in\left[s_{0}, T\right]$, является неподвижной точкой оператора $L$ на отрезке $[0, T]$. Последнее согласно теореме 1 означает, что $\widehat{G} \equiv G$. Следовательно, если $G^{\prime}\left(s_{0}\right)=0$, то $G^{\prime}(s)=0$ при $s>s_{0}$. Отсюда вытекает, что $G^{\prime}(t)>0$ при $G(t)<$ $\|G\|_{C\left(\mathbf{R}_{+}\right)}$. Это же рассуждение проходит в случае функции $F_{K}$, т.е. если $F_{K}^{\prime}\left(s_{0}\right)=0$, то $F_{K}^{\prime}(s)=0$ при $s>s_{0}$.

Перепишем определение меры $\mu$, используя условные математические ожидания с учетом равенства $\mathbf{P}\left(l_{1} \leqslant 0 \mid r_{1}=u\right)=0$ :

$$
\begin{aligned}
\mu([0, s] \times[0, t])= & a \mathbf{P}\left(\widehat{r}_{1} \leqslant t\right)+b \int_{0}^{(t-s) \vee 0} \Psi_{u}(G(s)) \mathbf{P}\left(r_{1} \in d u\right) \\
& +b \int_{(t-s) \vee 0}^{t} \Psi_{u}(G(t-u)) \mathbf{P}\left(r_{1} \in d u\right) .
\end{aligned}
$$

Заметим также, что из (2.2) вытекает соотношение $A_{1}^{K}=F_{K, 1}^{-1}\left(l_{1}\right)$, где функция $F_{K, 1}$ определятся аналогично $F_{K}$, только с заменой $\mathscr{Z}_{K}$ на оператор $\mathscr{Z}_{K, 1}: D(\mathbf{R}) \rightarrow D_{1}$, заданный равенством

$$
\mathscr{Z}_{K, 1} f(t):=\frac{1}{K} \sum_{i=1}^{a_{K} K} \mathbf{1}_{\left\{\hat{r}_{i}>t\right\}}+\frac{1}{K} \sum_{j=2}^{b_{K} K} \mathbf{1}_{\left\{f\left(t-r_{j}\right)<l_{j} \leqslant f(t)\right\}} .
$$

\section{5. Основные результаты и доказательства.}

Лемма 1. Пусть $X_{1}, \ldots, X_{n}$ - независимье одинаково распределенные случайнье величинь с функиией распределения $F$, a $F_{n}(t)=$ $n^{-1} \sum_{i=1}^{n} \mathbf{1}_{\left\{X_{i} \leqslant t\right\}}$ - эмпирическая функиия распределения. Тогда при $p \geqslant 1$ выполнено равенство

$$
\left(\mathbf{E}\left\|F-F_{n}\right\|_{C(\mathbf{R})}^{p}\right)^{1 / p} \leqslant n^{-1 / 2}(1+C(p) \sqrt{p}),
$$

где $C(p)=p^{-1 / 2}\left(\int_{1}^{+\infty} 2 p y^{p-1} e^{-2 y^{2}} d y\right)^{1 / p}$. Кроме того, $C(p)$ может быть выгбрано равнылм 0 в случае $p=1$ и 0.2 при $p>5$. 
Док аз а т ель с т в о. Согласно $[9], \mathbf{P}\left(\left\|F-F_{n}\right\|_{C(\mathbf{R})}>x\right) \leqslant$ $2 e^{-2 n x^{2}}$ для любых $x>0$. Тогда, используя соотношение $\mathbf{E}|\xi|^{p}=$ $\int_{0}^{+\infty} p x^{p-1} \mathbf{P}(|\xi|>x) d x$, имеем

$$
\begin{aligned}
\mathbf{E}\left\|F-F_{n}\right\|_{C(\mathbf{R})}^{p} & =\int_{0}^{+\infty} p x^{p-1} \mathbf{P}\left(\left\|F-F_{n}\right\|_{C(\mathbf{R})}>x\right) d x \\
& \leqslant \int_{0}^{\varepsilon} p x^{p-1} d x+\int_{\varepsilon}^{+\infty} 2 p x^{p-1} e^{-2 n x^{2}} d x \\
& =\varepsilon^{p}+n^{-p / 2} \int_{\varepsilon \sqrt{n}}^{+\infty} 2 p y^{p-1} e^{-2 y^{2}} d y .
\end{aligned}
$$

Полагая $\varepsilon=n^{-1 / 2}$, приходим к неравенству $\mathbf{E}\left\|F-F_{n}\right\|_{C(\mathbf{R})}^{p} \leqslant n^{-p / 2}(1+$ $\left.\int_{1}^{+\infty} 2 p y^{p-1} e^{-2 y^{2}} d y\right)$. Учитывая, что $\left(1+x^{p}\right)^{1 / p} \leqslant 1+x$ при $x \geqslant 0$ и $p \geqslant 1$, получаем искомую оценку (5.1).

Далее, используя формулу Стирлинга $\Gamma(x+1)=\sqrt{2 \pi x} x^{x} e^{r(x)-x}$, где $r(x) \leqslant 1 /(12 x), x>0$ (см., например, [10, с. 83]), при $p>2$ имеем

$$
\int_{1}^{+\infty} 2 p y^{p-1} e^{-2 y^{2}} d y \leqslant \frac{p}{2^{p / 2}} \Gamma\left(\frac{p}{2}\right) \leqslant \sqrt{e \pi} p\left(\frac{p-2}{4 e}\right)^{(p-1) / 2} e^{1 /(6 p-12)} .
$$

Последнее влечет неравенство $C(p) \leqslant A(p)$, где

$$
A(p)=\left(\frac{4 e^{2} \pi p^{2}}{p-2}\right)^{1 /(2 p)} e^{1 /(p(6 p-12))} \sqrt{\frac{p-2}{4 e p}},
$$

a $p>2$. Осталось заметить, что $A(p)<0.2$ при $p>5$.

При $p=1$ достаточно положить $\varepsilon=\sqrt{(2 n)^{-1} \ln 2}$, это влечет

$$
\begin{aligned}
\mathbf{E}\left\|F-F_{n}\right\|_{C(\mathbf{R})} & \leqslant \sqrt{\frac{\ln 2}{2 n}}+\frac{1}{\sqrt{n}} \int_{\sqrt{(\ln 2) / 2}}^{+\infty} 2 e^{-2 y^{2}} d y \\
& =\sqrt{\frac{\ln 2}{2 n}}+\sqrt{\frac{2 \pi}{n}}\left(1-\Phi_{\mathscr{N}(0,1)}(2 \ln 2)\right)<\frac{1}{\sqrt{n}},
\end{aligned}
$$

где $\Phi_{\mathscr{N}(0,1)}$ - функция распределения стандартной нормальной величины.

Лемма 2. Пусть $h(\cdot)=h(\cdot, \omega)$ есть случайный элемент со значениями в пространстве $C(\mathbf{R})$, причем $h(s)=0$ при $s \leqslant 0$, всюду существует производная $h^{\prime} u 0 \leqslant h^{\prime}(s)<\gamma$ для некоторого $\gamma>0$ и любого $s \in \mathbf{R}$. Тогда, если семейство случайньих величин $\left\{\left(l_{j}, r_{j}\right)\right\}_{j=1}^{n}$ удовлетворяет свойствам $1^{\circ}-5^{\circ}$, то найдутся такие константы $C_{1}>0 u$ $C_{2}>0$, что для всех $t \geqslant 0$

$$
\begin{aligned}
\mathbf{E} \| & \frac{1}{n} \sum_{j=1}^{n} \mathbf{1}_{\left\{l_{j} \leqslant h\left(\cdot-r_{j}\right)\right\}}-\mathbf{P}\left(l_{1} \leqslant h\left(\cdot-r_{1}\right)\right) \|_{C[0, t]} \\
& \leqslant \max \left\{\frac{4}{\sqrt{n}}+\left(C_{1}+2 \beta \gamma\right)\left(\frac{t}{n}\right)^{1 / 3}, \frac{C_{2} \beta \gamma+1}{\sqrt{n}}\right\},
\end{aligned}
$$


здесь $\mathbf{P}\left(l_{1} \leqslant h\left(\cdot-r_{1}\right)\right)(\omega)=\int_{\Omega} \mathbf{1}_{\left\{l_{1}(\tilde{\omega}) \leqslant h\left(\cdot-r_{1}(\tilde{\omega}), \omega\right)\right\}} \mathbf{P}(d \widetilde{\omega})$. Константьи $C_{1} u$ $C_{2}$ могут быть выбраны равными соответственно 0.4 и $7 \sqrt{7}$.

Д о к аз а т ел ь с т в о. Обозначим $I_{n, t}:=\| \frac{1}{n} \sum_{j=1}^{n} \mathbf{1}_{\left\{l_{j} \leqslant h\left(\cdot-r_{j}\right)\right\}}-$ $\mathbf{P}\left(l_{1} \leqslant h\left(\cdot-r_{1}\right)\right) \|_{C[0, t]}$. Фиксируем произвольное $\varepsilon>0$ такое, что $t / \varepsilon-$ целое число. Разобьем отрезок $[0, t]$ на $t / \varepsilon$ частей. Заметим, что из условия леммы следует, что $h(\varepsilon) \in[0, \gamma \varepsilon)$, и если $h(k \varepsilon) \in[l \gamma \varepsilon,(l+1) \gamma \varepsilon)$ для некоторых $k$ и $l$, то возможны два случая: $(*) h((k+1) \varepsilon) \in[l \gamma \varepsilon,(l+$ 1) $\gamma \varepsilon)$ и $(* *) h((k+1) \varepsilon) \in[(l+1) \gamma \varepsilon,(l+2) \gamma \varepsilon)$. Принимая это во внимание, рассмотрим следующее разложение:

$$
1=\mathbf{1}_{A_{1}}+\mathbf{1}_{A_{0}}=\mathbf{1}_{A_{00}}+\mathbf{1}_{A_{01}}+\mathbf{1}_{A_{10}}+\mathbf{1}_{A_{11}}=\cdots=\sum_{i=0}^{2^{t / \varepsilon-2}-1} \mathbf{1}_{A_{b(i)}},
$$

где $b(i)$ - двоичное представление числа $i$, состоящее из $t / \varepsilon-2$ цифр,

$$
\begin{aligned}
A_{0} & =\{h(2 \varepsilon) \in[0, \gamma \varepsilon)\}, \\
A_{1} & =\{h(2 \varepsilon) \in[\gamma \varepsilon, 2 \gamma \varepsilon)\}, \\
A_{00} & =\{h(2 \varepsilon) \in[0, \gamma \varepsilon), h(3 \varepsilon) \in[0, \gamma \varepsilon)\}, \\
A_{10} & =\{h(2 \varepsilon) \in[0, \gamma \varepsilon), h(3 \varepsilon) \in[\gamma \varepsilon, 2 \gamma \varepsilon)\}, \\
A_{01} & =\{h(2 \varepsilon) \in[\gamma \varepsilon, 2 \gamma \varepsilon), h(3 \varepsilon) \in[\gamma \varepsilon, 2 \gamma \varepsilon)\}, \\
A_{11} & =\{h(2 \varepsilon) \in[\gamma \varepsilon, 2 \gamma \varepsilon), h(3 \varepsilon) \in[2 \gamma \varepsilon, 3 \gamma \varepsilon)\}
\end{aligned}
$$

и т.д.: приписанной слева цифре 0 соответствует случай (*), а цифре 1 случай $(* *)$, т.е.

$$
A_{b(i)}=\left\{h(k \varepsilon) \in\left[S_{k-1}(i) \gamma \varepsilon,\left(S_{k-1}(i)+1\right) \gamma \varepsilon\right), k=2, \ldots, t / \varepsilon-1\right\},
$$

где $S_{k}(i)$ - сумма цифр до $k$-й в двоичном представлении числа $i$. Заметим, что при таком определении $A_{b(i)} A_{b(j)}=\varnothing$ для различных $i$ и $j$. Положим для краткости $A_{b(i)}=A_{i}$. Идея дальнейшего доказательства состоит в том, чтобы ограничить случайный элемент $h$ для любого события $A_{i}$ некоторыми неслучайными функциями сверху и снизу и перейти к оценке выражения в условии леммы для этих неслучайных функций. Для реализации сказанного определим при каждом $i=1, \ldots, 2^{t / \varepsilon-2}-1$ следующие непрерывные справа функции:

$$
\begin{aligned}
h_{i}^{1}(s) & :=\sum_{k=2}^{t / \varepsilon-1} S_{k-1}(i) \gamma \varepsilon \cdot \mathbf{1}_{[k \varepsilon,(k+1) \varepsilon)}(s)+S_{t / \varepsilon-2}(i) \gamma \varepsilon \cdot \mathbf{1}_{\{t\}}(s), \\
h_{i}^{2}(s) & :=h_{i}^{1}(s)+2 \gamma \varepsilon \mathbf{1}_{[0,+\infty]}(s)
\end{aligned}
$$

при $s \leqslant t$ и $h_{i}^{m}(s):=h_{i}^{m}(t)$ при $s>t, m=1,2$. Тогда в случае наступления события $A_{i}$ для любого $s \in[0, t]$ выполняются неравенства 
$h_{i}^{1}(s) \leqslant h(s) \leqslant h_{i}^{2}(s)$, влекущие

$$
\begin{gathered}
\mathbf{1}_{\left\{l_{j} \leqslant h_{i}^{1}\left(s-r_{j}\right)\right\}} \leqslant \mathbf{1}_{\left\{l_{j} \leqslant h\left(s-r_{j}\right)\right\}} \leqslant \mathbf{1}_{\left\{l_{j} \leqslant h_{i}^{2}\left(s-r_{j}\right)\right\}}, \\
\mathbf{P}\left(l_{1} \leqslant h_{i}^{1}\left(s-r_{1}\right)\right) \leqslant \mathbf{P}\left(l_{1} \leqslant h\left(s-r_{1}\right)\right) \leqslant \mathbf{P}\left(l_{1} \leqslant h_{i}^{2}\left(s-r_{1}\right)\right) .
\end{gathered}
$$

Поэтому на $A_{i}$

$$
\begin{aligned}
& \left|\frac{1}{n} \sum_{j=1}^{n} \mathbf{1}_{\left\{l_{j} \leqslant h\left(s-r_{j}\right)\right\}}-\mathbf{P}\left(l_{1} \leqslant h\left(s-r_{1}\right)\right)\right| \\
& \leqslant \max _{m=1,2}\left|\frac{1}{n} \sum_{j=1}^{n} \mathbf{1}_{\left\{l_{j} \leqslant h_{i}^{m}\left(s-r_{j}\right)\right\}}-\mathbf{P}\left(l_{1} \leqslant h_{i}^{m}\left(s-r_{1}\right)\right)\right| \\
& \quad+\mathbf{P}\left(l_{1} \leqslant h_{i}^{2}\left(s-r_{1}\right)\right)-\mathbf{P}\left(l_{1} \leqslant h_{i}^{1}\left(s-r_{1}\right)\right) .
\end{aligned}
$$

Оценим второе и третье слагаемые в правой части последнего неравенства. Используя условные математические ожидания, а также принимая во внимание свойство $5^{\circ}$ из п. 2 и неравенство $0 \leqslant h_{i}^{2}(s)-h_{i}^{1}(s) \leqslant 2 \gamma \varepsilon$, имеем

$$
\begin{aligned}
\mathbf{P}\left(l_{1}\right. & \left.\leqslant h_{i}^{2}\left(s-r_{1}\right)\right)-\mathbf{P}\left(l_{1} \leqslant h_{i}^{1}\left(t-r_{1}\right)\right) \\
& =\int_{0}^{+\infty}\left[\Psi_{u}\left(h_{i}^{2}(s-u)\right)-\Psi_{u}\left(h_{i}^{1}(s-u)\right)\right] \Phi(d u) \leqslant 2 \beta \gamma \varepsilon .
\end{aligned}
$$

$\mathrm{B}$ итоге на $A_{i}$ получаем

$$
I_{n, t} \leqslant \sum_{m=1,2}\left\|\frac{1}{n} \sum_{j=1}^{n} \mathbf{1}_{\left\{l_{j} \leqslant h_{i}^{m}\left(\cdot-r_{j}\right)\right\}}-\mathbf{P}\left(l_{1} \leqslant h_{i}^{m}\left(\cdot-r_{1}\right)\right)\right\|_{C[0, t]}+2 \beta \gamma \varepsilon .
$$

Следовательно, на всем вероятностном пространстве

$$
\begin{aligned}
\mathbf{E} I_{n, t} \leqslant & \sum_{i=0}^{2^{t / \varepsilon-2}-1} \sum_{m=1,2} \mathbf{E}\left\|\frac{1}{n} \sum_{j=1}^{n} \mathbf{1}_{\left\{l_{j} \leqslant h_{i}^{m}\left(\cdot-r_{j}\right)\right\}}-\mathbf{P}\left(l_{1} \leqslant h_{i}^{m}\left(\cdot-r_{1}\right)\right)\right\|_{C[0, t]} \mathbf{1}_{A_{i}} \\
& +2 \beta \gamma \varepsilon .
\end{aligned}
$$

Правая часть последнего соотношения, согласно неравенству Гёльдера, при $p>1$ оценивается сверху выражением

$$
\sum^{\prime}\left(\mathbf{E}\left\|\frac{1}{n} \sum_{j=1}^{n} \mathbf{1}_{\left\{l_{j} \leqslant h_{i}^{m}\left(\cdot-r_{j}\right)\right\}}-\mathbf{P}\left(l_{1} \leqslant h_{i}^{m}\left(\cdot-r_{1}\right)\right)\right\|_{C[0, t]}^{p}\right)^{1 / p}\left(\mathbf{P}\left(A_{i}\right)\right)^{1-1 / p}+2 \beta \gamma \varepsilon,
$$

которое можно переписать в виде

$$
\begin{gathered}
\sum^{\prime}\left(\mathbf{E}\left\|\frac{1}{n} \sum_{j=1}^{n} \mathbf{1}_{\left\{\left(h_{i}^{m}\right)^{-1}\left(l_{j}\right)+r_{j} \leqslant \cdot\right\}}-\mathbf{P}\left(\left(h_{i}^{m}\right)^{-1}\left(l_{1}\right)+r_{1} \leqslant \cdot\right)\right\|_{C[0, t]}^{p}\right)^{1 / p} \\
\times\left(\mathbf{P}\left(A_{i}\right)\right)^{1-1 / p}+2 \beta \gamma \varepsilon
\end{gathered}
$$


здесь $\sum^{\prime}$ обозначает суммирование по всем $i$ и $m$, фигурирующим в (5.2). По лемме 1 сумма, полученная таким образом, ограничивается сверху выражением

$$
2 n^{-1 / 2}(1+C(p) \sqrt{p}) \sum_{i=0}^{2^{t / \varepsilon-2}-1}\left(\mathbf{P}\left(A_{i}\right)\right)^{1-1 / p}+2 \beta \gamma \varepsilon,
$$

если в этой лемме взять $X_{j}=\left(h_{i}^{m}\right)^{-1}\left(l_{j}\right)+r_{j}$. Замечая, что $\sum_{i=0}^{2^{t / \varepsilon-2}-1} \mathbf{P}\left(A_{i}\right)=1$ и максимум выражения $\sum_{i=1}^{I} x_{i}^{\alpha}$ при условиях $0<$ $\alpha<1, \sum_{i=1}^{I} x_{i}=1$ и $x_{i} \geqslant 0$ достигается на значениях $x_{i}=1 / I$ $(i=1, \ldots, I)$, приходим к неравенству

$$
\begin{gathered}
2 n^{-1 / 2}(1+C(p) \sqrt{p}) \sum_{i=0}^{2^{t / \varepsilon-2}-1}\left(\mathbf{P}\left(A_{i}\right)\right)^{1-1 / p}+2 \beta \gamma \varepsilon \\
\leqslant 2 n^{-1 / 2}(1+C(p) \sqrt{p})\left(2^{t / \varepsilon-2}\right)^{1 / p}+2 \beta \gamma \varepsilon .
\end{gathered}
$$

Для завершения доказательства осталось подходящим образом выбрать параметры $\varepsilon$ и $p$. Полагая $p=t / \varepsilon-2$, имеем

$$
2 n^{-1 / 2}(1+C(p) \sqrt{p})\left(2^{t / \varepsilon-2}\right)^{1 / p} \leqslant 4 n^{-1 / 2}\left(1+C\left(\varepsilon^{-1} t-2\right) \sqrt{\varepsilon^{-1} t-2}\right) .
$$

Оптимизируя по $\varepsilon$, выберем $\varepsilon=t /\left\lceil n^{1 / 3} t^{2 / 3}\right\rceil$, здесь $\lceil x\rceil-$ минимальное целое число, не меньшее $x$. Тогда, используя неравенства $\sqrt{t / \varepsilon-2}=$ $\sqrt{\left\lceil n^{1 / 3} t^{2 / 3}\right\rceil-2} \leqslant n^{1 / 6} t^{1 / 3}, t /\left\lceil n^{1 / 3} t^{2 / 3}\right\rceil \leqslant(t / n)^{1 / 3}$, окончательно получаем

$$
\mathbf{E} I_{n, t} \leqslant 4 n^{-1 / 2}+\left(4 C\left(\left\lceil n^{1 / 3} t^{2 / 3}\right\rceil-2\right)+2 \beta \gamma\right)\left(\frac{t}{n}\right)^{1 / 3} .
$$

Заметим, что верхнюю оценку рассматриваемого выражения можно получить другим путем. А именно, если $t \leqslant C_{2} / \sqrt{n}$ для некоторого $C_{2}>0$, то на отрезке $[0, t]$ выполняются неравенства $0 \leqslant h(\cdot) \leqslant \gamma t \leqslant$ $C_{2} \gamma / \sqrt{n}$, поэтому аналогично предыдущему, с учетом леммы 1 и строгой положительности $l_{j}$, имеем

$$
\begin{aligned}
\mathbf{E} I_{n, t} & \leqslant \mathbf{E}\left|\frac{1}{n} \sum_{j=1}^{n} \mathbf{1}_{\left\{l_{j} \leqslant C_{2} \gamma / \sqrt{n}\right\}}-\mathbf{P}\left(l_{1} \leqslant \frac{C_{2} \gamma}{\sqrt{n}}\right)\right|+\mathbf{P}\left(l_{1} \leqslant \frac{C_{2} \gamma}{\sqrt{n}}\right)-\mathbf{P}\left(l_{1} \leqslant 0\right) \\
& \leqslant \mathbf{E}\left|\frac{1}{n} \sum_{j=1}^{n} \mathbf{1}_{\left\{l_{j} \leqslant C_{2} \gamma / \sqrt{n}\right\}}-\mathbf{P}\left(l_{1} \leqslant \frac{C_{2} \gamma}{\sqrt{n}}\right)\right|+\frac{C_{2} \gamma \beta}{\sqrt{n}} \leqslant \frac{C_{2} \beta \gamma+1}{\sqrt{n}} .
\end{aligned}
$$

Положив $C_{2}=7 \sqrt{7}$, искомую оценку можно получить, комбинируя предыдущие неравенства и учитывая, что по лемме 1 выполнено неравенство $C\left(\left\lceil n^{1 / 3} t^{2 / 3}\right\rceil-2\right) \leqslant 0.2=C_{1}$ при $t>7 \sqrt{7} / \sqrt{n}$.

Нам потребуется следующий элементарный вариант леммы Гронуолла-Беллмана. 
Лемма 3. Пусть $f(t)$ - непрерыьвная функция на $[0, T]$, удовлетворяющая неравенству $f(t) \leqslant q \int_{0}^{t} f(s) d s$ при $t \in[0, T]$ с некоторой константой $q \geqslant 0$. Тогда $f(t) \leqslant 0$ при $t \in[0, T]$.

Д о к а з а т е л ь с т в о. Утверждение вытекает из следующей цепочки неравенств:

$$
\begin{aligned}
f(t) & \leqslant q \int_{0}^{t} f(s) d s \leqslant q^{2} \int_{0}^{t} \int_{0}^{t_{1}} f(s) d s d t_{1} \leqslant \cdots \\
& \leqslant q^{n+1} \int_{0}^{t} \int_{0}^{t_{1}} \cdots \int_{0}^{t_{n}} f(s) d s d t_{n} \cdots d t_{1} \\
& \leqslant q^{n+1}\|f\|_{C[0, T]} \int_{0}^{t} \int_{0}^{t_{1}} \cdots \int_{0}^{t_{n}} d s d t_{n} \cdots d t_{1}=\frac{(q t)^{n+1}}{(n+1) !}\|f\|_{C[0, T]} \rightarrow 0
\end{aligned}
$$

при $n \rightarrow \infty$.

Теорема 3. Пусть выполнень свойства $1^{\circ}-5^{\circ}$ и $\lambda(t, x)$ удовлетворяет условиям 1-4. Тогда для любых $T \in \mathbf{R}_{+}$и для всех $H \in \mathscr{F}$

$\left|\mathbf{E} H\left(\xi_{K}^{T}\right)-\mathbf{E} H\left(\mu^{T}\right)\right| \leqslant \frac{\sqrt{a_{K}}+\sqrt{b_{K}}}{\sqrt{K}}+\frac{2}{K}+\alpha \beta b_{K} S(K) \int_{0}^{T} h_{0}(T-u) \Phi(d u)$,

где $h_{0}(t)=h_{1}(t, \alpha \beta b(1+\Phi(t))), h_{1}(t, q)=\left(e^{q t}-1-q t\right) / q^{2}-$ $\left(e^{q t}-1\right) / q, S(K)=\sqrt{a_{K}} / \sqrt{K}+3 / K+\max \left\{5 \sqrt{b_{K}} / \sqrt{K}+\left(C_{1}+\right.\right.$ $\left.2 \beta \gamma) b_{K}^{2 / 3}(T / K)^{1 / 3}, \sqrt{b_{K}}\left(C_{2} \beta \gamma+2\right) / \sqrt{K}\right\}$, а константь $C_{1}$ и $C_{2}$ могут быть выбраны равныли соответственно 0.4 и $7 \sqrt{7}$.

Д о к а з а т е л ь с т в о. По определению класса $\mathscr{F}$ найдутся такие $f, m$ и $\phi_{k}(k=1, \ldots, m)$, что выполнено равенство $\mathbf{E} H(\nu)=$ $\mathbf{E} f\left(\left\langle\nu, \phi_{k}\right\rangle, k=1, \ldots, m\right)$. Пользуясь для гладких $h$ соотношением $\mathbf{E} h(W)-\mathbf{E} h(Z)=\int_{0}^{1} \mathbf{E} h^{\prime}(\theta W+(1-\theta) Z)(W-Z) d \theta$, имеем

$$
\begin{aligned}
\mathbf{E} H\left(\xi_{K}^{T}\right)-\mathbf{E} H\left(\mu^{T}\right)=\int_{0}^{1} & \sum_{j=1}^{m} \mathbf{E} f_{x_{j}}^{\prime}\left(\left\langle\theta \xi_{K}^{T}+(1-\theta) \mu^{T}, \phi_{k}\right\rangle, k=1, \ldots, m\right) \\
& \times\left\langle\xi_{K}^{T}-\mu^{T}, \phi_{j}\right\rangle d \theta .
\end{aligned}
$$

Так как интегрирование ведется по отрезку единичной длины, из оценки $\sum_{j=1}^{m}\left\|f_{x_{j}}^{\prime}\right\| \leqslant 1$ получаем неравенство

$$
\left|\mathbf{E} H\left(\xi_{K}^{T}\right)-\mathbf{E} H\left(\mu^{T}\right)\right| \leqslant \sup _{\phi \in C_{b}^{\infty}\left(\mathbf{R}^{2}\right):\|\phi\| \leqslant 1,\left\|\phi^{\prime}\right\| \leqslant 1} \mathbf{E}\left|\left\langle\xi_{K}^{T}-\mu^{T}, \phi\right\rangle\right| .
$$

Для любого фиксированного $\phi$ оценим выражение, стоящее под знаком супремума. По определению $\xi_{k}^{T}$ и $\mu^{T}$ имеем

$$
\begin{aligned}
\left\langle\xi_{K}^{T}, \phi\right\rangle & =\frac{1}{K} \sum_{i=1}^{a_{K} K} \phi\left(0, \widehat{r}_{i}\right) \mathbf{1}_{\left\{\hat{r}_{i} \leqslant T\right\}}+\frac{1}{K} \sum_{j=1}^{b_{K} K} \phi\left(A_{j}^{K}, A_{j}^{K}+r_{j}\right) \mathbf{1}_{\left\{A_{j}^{K}+r_{j} \leqslant T\right\}}, \\
\left\langle\mu^{T}, \phi\right\rangle & =a \mathbf{E} \phi\left(0, \widehat{r}_{1}\right) \mathbf{1}_{\left\{\hat{r}_{1} \leqslant T\right\}}+b \mathbf{E} \phi\left(G^{-1}\left(l_{1}\right), G^{-1}\left(l_{1}\right)+r_{1}\right) \mathbf{1}_{\left\{G^{-1}\left(l_{1}\right)+r_{1} \leqslant T\right\}} .
\end{aligned}
$$


Обозначим для удобства

$$
\begin{aligned}
X_{j} & :=\phi\left(A_{j}^{K}, A_{j}^{K}+r_{j}\right) \mathbf{1}_{\left\{A_{j}^{K}+r_{j} \leqslant T\right\}}, \\
Y_{j} & :=\phi\left(G^{-1}\left(l_{j}\right), G^{-1}\left(l_{j}\right)+r_{j}\right) \mathbf{1}_{\left\{G^{-1}\left(l_{j}\right)+r_{j} \leqslant T\right\}} .
\end{aligned}
$$

Добавляя и вычитая выражение $K^{-1} \sum_{j=1}^{b_{K} K} Y_{j}$, затем группируя слагаемые и учитывая, что $\|\phi\| \leqslant 1,\left|a K-a_{K} K\right| \leqslant 1,\left|b K-b_{K} K\right| \leqslant 1$, получаем неравенство

$$
\begin{aligned}
\mathbf{E}\left|\left\langle\xi_{K}^{T}-\mu^{T}, \phi\right\rangle\right| \leqslant & a_{K} \mathbf{E}\left|\frac{1}{a_{K} K} \sum_{i=1}^{a_{K} K} \phi\left(0, \widehat{r}_{i}\right) \mathbf{1}_{\left\{\hat{r}_{i} \leqslant T\right\}}-\mathbf{E} \phi\left(0, \widehat{r}_{1}\right) \mathbf{1}_{\left\{\hat{r}_{1} \leqslant T\right\}}\right| \\
& +\frac{1}{K} \mathbf{E}\left|\sum_{j=1}^{b_{K} K} X_{j}-\sum_{j=1}^{b_{K} K} Y_{j}\right| \\
& +b_{K} \mathbf{E}\left|\frac{1}{b_{K} K} \sum_{j=1}^{b_{K} K} Y_{j}-\mathbf{E} Y_{1}\right|+\frac{2}{K} .
\end{aligned}
$$

Под знаками первого и третьего математических ожиданий в правой части последней формулы стоят суммы н.о.р. случайных величин, так как $\left\{\widehat{r}_{i}\right\}_{i=1}^{a_{K} K}-$ н.о.p. и $\left\{\left(r_{j}, l_{j}\right)\right\}_{j=1}^{b_{K} K}-$ н.о.р. Тогда в силу неравенств $\left(\mathbf{E}\left|n^{-1} \sum_{i=1}^{n} V_{i}-\mathbf{E} V_{1}\right|\right)^{2} \leqslant \mathbf{D}\left(n^{-1} \sum_{i=1}^{n} V_{i}\right)=n^{-1} \mathbf{D} V_{1} \leqslant n^{-1}$, справедливых, когда $\left\{V_{i}\right\}_{i=1}^{n}$ - н.о.р. и $\left|V_{i}\right| \leqslant 1$ п.н., заключаем, что

$$
\mathbf{E}\left|\left\langle\xi_{K}^{T}-\mu^{T}, \phi\right\rangle\right| \leqslant \frac{a_{K}}{\sqrt{a_{K} K}}+\frac{b_{K}}{\sqrt{b_{K} K}}+\frac{2}{K}+\frac{1}{K} \mathbf{E}\left|\sum_{j=1}^{b_{K} K} X_{j}-\sum_{j=1}^{b_{K} K} Y_{j}\right| .
$$

Из того, что $\left\{\widehat{r}_{i}\right\}_{i=1}^{a_{K} K}-$ н.о.р. и $\left\{\left(r_{j}, l_{j}\right)\right\}_{j=1}^{b_{K} K}-$ н.о.р., следует одинаковая распределенность случайных векторов $\left(X_{j}, Y_{j}\right)$ при всех $j$, откуда

$$
\frac{1}{K} \mathbf{E}\left|\sum_{j=1}^{b_{K} K} X_{j}-\sum_{j=1}^{b_{K} K} Y_{j}\right| \leqslant b_{K} \mathbf{E}\left|X_{1}-Y_{1}\right| .
$$

Поскольку $A_{1}^{K} \geqslant 0$ п.н. и $G^{-1}\left(l_{1}\right) \geqslant 0$ п.н., то при $r_{1}>T$ индикаторы в определении $X_{1}$ и $Y_{1}$ обращаются в нуль, поэтому и сами $X_{1}$ и $Y_{1}$ обращаются в нуль. Учитывая сказанное и переходя к условным математическим ожиданиям, получаем равенство

$$
\mathbf{E}\left|X_{1}-Y_{1}\right|=\mathbf{E}\left\{\mathbf{E}\left(\left|X_{1}-Y_{1}\right| \mid r_{1}\right)\right\}=\int_{0}^{T} \mathbf{E}\left(\left|X_{1}-Y_{1}\right| \mid r_{1}=u\right) \mathbf{P}\left(r_{1} \in d u\right) .
$$

Так как $A_{1}^{K}=F_{K, 1}^{-1}\left(l_{1}\right)$ (см. п. 4), где $F_{K, 1}(t)-$ некоторая функция от случайных элементов $\left\{\left(r_{j}, l_{j}\right)\right\}_{j=2}^{b_{K} K}$ и $\left\{\widehat{r}_{i}\right\}_{i=1}^{a_{K} K}$, не зависящих от $r_{1}$, то $\mathbf{E}\left(\left|X_{1}-Y_{1}\right| \mid r_{1}=u\right)=\mathbf{E}\left|X_{1}^{u}-Y_{1}^{u}\right|$, где

$$
\begin{aligned}
X_{1}^{u} & :=\phi\left(F_{K, 1}^{-1}\left(l_{1}^{u}\right), F_{K, 1}^{-1}\left(l_{1}^{u}\right)+u\right) \mathbf{1}_{\left\{F_{K, 1}^{-1}\left(l_{1}^{u}\right)+u \leqslant T\right\}}, \\
Y_{1}^{u} & :=\phi\left(G^{-1}\left(l_{1}^{u}\right), G^{-1}\left(l_{1}^{u}\right)+u\right) \mathbf{1}_{\left\{G^{-1}\left(l_{1}^{u}\right)+u \leqslant T\right\}} .
\end{aligned}
$$


Здесь случайная величина $l_{1}^{u}$ имеет такое же распределение, как $l_{1}$, при фиксированном $u$ и не зависит от семейств случайных величин $\left\{\left(r_{j}, l_{j}\right)\right\}_{j=2}^{b_{K} K}$ и $\left\{\widehat{r}_{i}\right\}_{i=1}^{a_{K} K}$.

Пусть $\varphi(t):=\phi(t, t+u), B:=\left\{l_{1}^{u} \leqslant F_{K, 1}(T-u)\right\}, C:=\left\{l_{1}^{u} \leqslant G(T-u)\right\}$. Используя введенные обозначения, имеем

$$
\mathbf{E}\left|X_{1}^{u}-Y_{1}^{u}\right|=\mathbf{E}\left|\varphi\left(F_{K, 1}^{-1}\left(l_{1}^{u}\right)\right) \mathbf{1}_{B}-\varphi\left(G^{-1}\left(l_{1}^{u}\right)\right) \mathbf{1}_{C}\right| .
$$

Из того, что $\mathbf{1}_{B}=\mathbf{1}_{B C}+\mathbf{1}_{B \bar{C}}, \mathbf{1}_{C}=\mathbf{1}_{B C}+\mathbf{1}_{\bar{B} C}$ и $\|\varphi\| \leqslant 1$, вытекает неравенство

$$
\begin{aligned}
& \mathbf{E}\left|\varphi\left(F_{K, 1}^{-1}\left(l_{1}^{u}\right)\right) \mathbf{1}_{B}-\varphi\left(G^{-1}\left(l_{1}^{u}\right)\right) \mathbf{1}_{C}\right| \\
& \quad \leqslant \mathbf{E} \mathbf{1}_{B \bar{C}}+\mathbf{E} \mathbf{1}_{\bar{B} C}+\mathbf{E}\left|\varphi\left(F_{K, 1}^{-1}\left(l_{1}^{u}\right)\right)-\varphi\left(G^{-1}\left(l_{1}^{u}\right)\right)\right| \mathbf{1}_{B C} .
\end{aligned}
$$

Оценим два первых слагаемых в правой части (5.9). Из независимости $l_{1}^{u}$ от $\left\{\left(r_{j}, l_{j}\right)\right\}_{j=2}^{b_{K} K}$ и $\left\{\widehat{r}_{i}\right\}_{i=1}^{a_{K} K}$, а также ограниченности $\psi_{u}$ следует, что

$$
\begin{aligned}
\mathbf{E} \mathbf{1}_{B \bar{C}}+\mathbf{E} \mathbf{1}_{\bar{B} C}= & \mathbf{E} \mathbf{1}_{\left\{G(T-u)<l_{1}^{u} \leqslant F_{K, 1}(T-u)\right\}}+\mathbf{E} \mathbf{1}_{\left\{F_{K, 1}(T-u)<l_{1}^{u} \leqslant G(T-u)\right\}} \\
\leqslant & \mathbf{E} \int_{G(T-u)}^{F_{K, 1}(T-u)} \psi_{u}(x) d x \mathbf{1}_{\left\{F_{K, 1}(T-u)>G(T-u)\right\}} \\
& +\mathbf{E} \int_{F_{K, 1}(T-u)}^{G(T-u)} \psi_{u}(x) d x \mathbf{1}_{\left\{F_{K, 1}(T-u)<G(T-u)\right\}} \\
\leqslant & \beta \mathbf{E}\left|F_{K, 1}(T-u)-G(T-u)\right| .
\end{aligned}
$$

Для оценки оставшегося слагаемого в (5.9) положим $g(t)=F_{K, 1}(t) \wedge G(t)$, тогда $\mathbf{1}_{B C}=\mathbf{1}_{[0, g(T-u))}\left(l_{1}^{u}\right)$. Неравенство $\left\|\varphi^{\prime}\right\| \leqslant 1$ и теорема Лагранжа влекут

$$
\begin{aligned}
& \mathbf{E}\left|\varphi\left(F_{K, 1}^{-1}\left(l_{1}^{u}\right)\right)-\varphi\left(G^{-1}\left(l_{1}^{u}\right)\right)\right| \mathbf{1}_{B C} \leqslant \mathbf{E}\left|F_{K, 1}^{-1}\left(l_{1}^{u}\right)-G^{-1}\left(l_{1}^{u}\right)\right| \mathbf{1}_{B C} \\
& =\mathbf{E}\left|\int_{G^{-1}\left(l_{1}^{u}\right)}^{F_{K, 1}^{-1}\left(l_{1}^{u}\right)} d s\right| \mathbf{1}_{[0, g(T-u))}\left(l_{1}^{u}\right)=\mathbf{E} \int_{0}^{g(T-u)}\left|\int_{G^{-1}(t)}^{F_{K, 1}^{-1}(t)} d s\right| \psi_{u}(t) d t .
\end{aligned}
$$

Раскроем знак модуля под интегралом в (5.11), тогда последнее выражение равно

$$
\begin{aligned}
\mathbf{E} \int_{0}^{g(T-u)} \int_{G^{-1}(t)}^{F_{K, 1}^{-1}(t)} d s \mathbf{1}_{\left\{F_{K, 1}^{-1}(t)>G^{-1}(t)\right\}} \psi_{u}(t) d t \\
\quad+\mathbf{E} \int_{0}^{g(T-u)} \int_{F_{K, 1}^{-1}(t)}^{G^{-1}(t)} d s \mathbf{1}_{\left\{F_{K, 1}^{-1}(t) \leqslant G^{-1}(t)\right\}} \psi_{u}(t) d t .
\end{aligned}
$$

Переставляя пределы интегрирования, а также учитывая равенства $F_{K, 1}^{-1}(0)=G^{-1}(0)=0$, замечание 1 и неотрицательность подынтегральных функций, последнюю сумму можно оценить сверху выражением

$$
\mathbf{E} \int_{0}^{F_{K, 1}^{-1}(g(T-u))} \int_{F_{K, 1}(s)}^{G(s)} \mathbf{1}_{\left\{F_{K, 1}^{-1}(t)>G^{-1}(t)\right\}} \psi_{u}(t) d t d s
$$




$$
+\mathbf{E} \int_{0}^{G^{-1}(g(T-u))} \int_{G(s)}^{F_{K, 1}(s)} \mathbf{1}_{\left\{F_{K, 1}^{-1}(t) \leqslant G^{-1}(t)\right\}} \psi_{u}(t) d t d s .
$$

Полученная сумма, в силу определения $g(t)$, свойства $5^{\circ}$ п. 2 и монотонности $F_{K, 1}$ и $G$, ограничивается сверху следующим образом:

$$
\begin{aligned}
& \beta \mathbf{E} \int_{0}^{T-u}\left|\int_{F_{K, 1}(s)}^{G(s)}\right| \mathbf{1}_{\left\{F_{K, 1}^{-1}(t)>G^{-1}(t)\right\}}-\mathbf{1}_{\left\{F_{K, 1}^{-1}(t) \leqslant G^{-1}(t)\right\}}|d t| d s \\
& \quad \leqslant \beta \mathbf{E} \int_{0}^{T-u}\left|F_{K, 1}(s)-G(s)\right| d s .
\end{aligned}
$$

Заметим, что $\mathbf{E}\left|F_{K, 1}(t)-G(t)\right| \leqslant \mathbf{E}\left\|F_{K, 1}-G\right\|_{C[0, t]}$, поэтому для оценки правой части неравенства (5.9) достаточно ограничить сверху $\mathbf{E}\left\|F_{K, 1}-G\right\|_{C[0, t]}$. Реализуем сказанное. Из справедливости равенств

$F_{K, 1}(t)-G(t)=L_{K, 1} F_{K, 1}(t)-L G(t)=L_{K, 1} F_{K, 1}(t)-L F_{K, 1}(t)+L F_{K, 1}-L G(t)$

следует, что для любого $t \in[0, T-u]$

$$
\mathbf{E}\left\|F_{K, 1}-G\right\|_{C[0, t]} \leqslant \mathbf{E}\left\|L_{K, 1} F_{K, 1}-L F_{K, 1}\right\|_{C[0, t]}+\mathbf{E}\left\|L F_{K, 1}-L G\right\|_{C[0, t]} .
$$

Оценим первое слагаемое в правой части (5.12). В силу липшицевости функционала $\lambda$ получаем

$$
\left\|L_{K, 1} F_{K, 1}-L F_{K, 1}\right\|_{C[0, t]} \leqslant \alpha \int_{0}^{t}\left\|\mathscr{Z}_{K, 1} F_{K, 1}-\mathscr{Z} F_{K, 1}\right\|_{C[0, s]} d s
$$

Рассмотрим подынтегральное выражение в последнем неравенстве. Так как $\left|a-a_{K}\right|=\left|b-b_{K}\right| \leqslant 1 / K$, то по определению операторов $\mathscr{Z}_{K, 1}$ и $\mathscr{Z}$ имеем

$\left\|\mathscr{Z}_{K, 1} F_{K, 1}-\mathscr{Z} F_{K, 1}\right\|_{C[0, s]} \leqslant \frac{3}{K}+a_{K} R_{1}+b_{K} \frac{b_{K} K-1}{b_{K} K} R_{2}+b_{K} \frac{b_{K} K-1}{b_{K} K} R_{3}$, где

$$
\begin{aligned}
R_{1} & :=\left\|\frac{1}{a_{K} K} \sum_{i=1}^{a_{K} K} \mathbf{1}_{\left\{\hat{r}_{i}>\cdot\right\}}-\mathbf{P}\left(\widehat{r}_{1}>\cdot\right)\right\|_{C[0, s]}, \\
R_{2} & :=\left\|\frac{1}{b_{K} K-1} \sum_{j=2}^{b_{K} K} \mathbf{1}_{\left\{F_{K, 1}^{-1}\left(l_{j}\right) \leqslant \cdot\right\}}-\mathbf{P}\left(F_{K, 1}^{-1}\left(l_{1}\right) \leqslant \cdot\right)\right\|_{C[0, s]}, \\
R_{3} & :=\left\|\frac{1}{b_{K} K-1} \sum_{j=2}^{b_{K} K} \mathbf{1}_{\left\{l_{j} \leqslant F_{K, 1}\left(\cdot-r_{j}\right)\right\}}-\mathbf{P}\left(l_{1} \leqslant F_{K, 1}\left(\cdot-r_{1}\right)\right)\right\|_{C[0, s]} .
\end{aligned}
$$

Из последней оценки и лемм 1 и 2 вытекает неравенство

$$
\mathbf{E}\left\|\mathscr{Z}_{K, 1} F_{K, 1}-\mathscr{Z} F_{K, 1}\right\|_{C[0, s]} \leqslant S(K),
$$


где $S(K)$ фигурирует в (5.3). Последнее позволяет оценить первое слагаемое в правой части (5.12). А именно, при $t \in[0, T]$ имеем

$$
\mathbf{E}\left\|L_{K, 1} F_{K, 1}-L F_{K, 1}\right\|_{C[0, t]} \leqslant \alpha S(K) t .
$$

Далее, используя определение оператора $L$, равенства $(4.1)$ и $\Psi^{\prime}(\cdot)=$ $\int_{\mathbf{R}} \psi_{v}(\cdot) \Phi(d v)$, а также липшицевость $\lambda$, оценим $\mathbf{E}\left\|L F_{K, 1}-L G\right\|_{C[0, t]}$. Для этого заметим, что

$$
\begin{aligned}
& \left|L F_{K, 1}(t)-L G(t)\right| \leqslant \alpha \int_{0}^{t}\left\|\mathscr{Z} F_{K, 1}-\mathscr{Z} G\right\|_{C[0, s]} d s \\
& \leqslant \alpha b \int_{0}^{t} \| \Psi\left(F_{K, 1}(\cdot)\right)-\Psi(G(\cdot))-\int_{0}^{\cdot}\left(\Psi_{v}\left(F_{K, 1}(\cdot-v)\right)\right. \\
& \left.\quad-\Psi_{v}(G(\cdot-v))\right) \Phi(d v) \|_{C[0, s]} d s \\
& \leqslant \alpha \beta b(1+\Phi(t)) \int_{0}^{t}\left\|F_{K, 1}-G\right\|_{C[0, s]} d s .
\end{aligned}
$$

Собирая оценки (5.12)-(5.14), приходим для $t \in[0, T-u]$ к неравенству

$\mathbf{E}\left\|F_{K, 1}-G\right\|_{C[0, t]} \leqslant \alpha S(K) t+\alpha \beta b(1+\Phi(T-u)) \int_{0}^{t} \mathbf{E}\left\|F_{K, 1}-G\right\|_{C[0, s]} d s$.

Докажем вспомогательное утверждение: если гладкая функция $y$ удовлетворяет неравенству $y^{\prime}(t) \leqslant p t+q y(t)$ при некоторых $p, q>0$ для любого $t$ из $\left[0, T^{\prime}\right]$ и краевому условию $y(0)=0$, то $y^{\prime}(t) \leqslant y_{\max }^{\prime}(t)$ и $y(t) \leqslant y_{\max }(t)$ на $\left[0, T^{\prime}\right]$, где $y_{\max }-$ решение дифференциального уравнения $y_{\max }^{\prime}(t)=p t+q y_{\max }(t)$ с краевым условием $y_{\max }(0)=0$, т.е. $y_{\max }(t)=p q^{-2}\left(e^{q t}-1-q t\right)$. Действительно, положив $f(t)=y^{\prime}(t)-y_{\max }^{\prime}(t)$ в лемме 3 , имеем неравенство $y^{\prime}(t) \leqslant y_{\max }^{\prime}(t)$ при $t \in\left[0, T^{\prime}\right]$, интегрируя которое, получаем $y(t) \leqslant y_{\max }(t)$.

Принимая во внимание доказанное утверждение и (5.15), приходим к неравенствам

$$
\begin{aligned}
\int_{0}^{t} \mathbf{E}\left\|F_{K, 1}-G\right\|_{C[0, s]} d s & \leqslant \frac{\alpha S(K)}{q^{2}}\left(e^{q t}-1-q t\right), \\
\mathbf{E}\left\|F_{K, 1}-G\right\|_{C[0, t]} & \leqslant \frac{\alpha S(K)}{q}\left(e^{q t}-1\right),
\end{aligned}
$$

где $q=\alpha \beta b(1+\Phi(T-u))$. Комбинируя оценки (5.4)-(5.16), получаем итоговый результат.

3 а м е ч а н и е 2 . Из теорем 1 и 3 следует, что $\operatorname{Law}\left(\xi_{K}^{T}\right) \stackrel{w}{\Longrightarrow} \delta_{\mu^{T}}$ при $K \rightarrow \infty$ (слабый закон больших чисел для процессов эпидемий). 
3 а м е ч а н и е 3. Основная сложность доказательства теоремы состоит в установлении леммы 2. При этом мы могли бы поступить иначе:

$$
\begin{aligned}
\mathbf{E} \| & \frac{1}{n} \sum_{j=1}^{n} \mathbf{1}_{\left\{l_{j} \leqslant h\left(\cdot-r_{j}\right)\right\}}-\mathbf{P}\left(l_{1} \leqslant h\left(\cdot-r_{1}\right)\right) \|_{C[0, t]} \\
& \leqslant \mathbf{E} \sup _{A \in \mathscr{A}}\left\|\frac{1}{n} \sum_{j=1}^{n} \mathbf{1}_{\left\{\left(l_{j}, r_{j}\right) \in A\right\}}-\mathbf{P}\left(\left(l_{1}, r_{1}\right) \in A\right)\right\|_{C[0, t]},
\end{aligned}
$$

где $\mathscr{A}=\{\{(x, y) \mid x \leqslant f(y), f$ - дифференцируемая функция с $-\gamma<$ $\left.\left.f^{\prime} \leqslant 0\right\}\right\}$. Существуют оценки подобных величин для систем выпуклых множеств для классов, допускающие комбинаторную характеризацию Вапника-Червоненкиса (см. [11], [12]). Однако в данном случае класс $\mathscr{A}$ устроен иначе, поэтому приходится получать оценку непосредственно.

3 а м е ч а н и е 4. Используя теорему 3 , нетрудно получить верхнюю оценку величины $\left|\mathbf{E} H\left(\xi_{K}^{T}\right)-\mathbf{E} H\left(\mu^{T}\right)\right|$, не зависящую от функции распределения $\Phi(\cdot)$, заменив интеграл в правой части (5.3) на максимум подынтегрального выражения на промежутке интегрирования.

3 а м е ч а н и е 5. Слагаемое в правой части (5.3), содержащее $h_{0}$, можно улучшить при больших $T$, например, в случае более сильного условия $2^{\prime}$ на функционал $\lambda$. Это показывает следующий результат.

Теорема 4. Пусть выполнень свойства $1^{\circ}-5^{\circ}$ и $\lambda(t, x)$ удовлетворяет условиям $1,2^{\prime}, 3,4$. Тогда для любых $T \in \mathbf{R}_{+}$и для всех $H \in \mathscr{F}$

$$
\begin{aligned}
\left|\mathbf{E} H\left(\xi_{K}^{T}\right)-\mathbf{E} H\left(\mu^{T}\right)\right| \leqslant & \frac{\sqrt{a_{K}}+\sqrt{b_{K}}}{\sqrt{K}}+\alpha \beta b_{K} S(K) \\
& \times \int_{0}^{T}\left(T-u+\frac{g_{1}\left(g_{0}(T-u)(T-u)\right)}{g_{0}(T-u)}\right. \\
& \left.\quad+(T-u) \frac{g_{1}^{\prime}\left(g_{0}(T-u)(T-u)\right)}{g_{0}(T-u)}\right) \Phi(d u),
\end{aligned}
$$

где $g_{0}(t)=\alpha \beta b(1+\Phi(t)), g_{1}(t)=\sum_{n=2}^{+\infty} t^{n} /(n !)^{2}, a a_{K}, b_{K}, S(K), C_{1} u C_{2}$ те же, ито и в теореме 3 .

Д о к а з а т е л ь с т в о. В силу неравенства $t^{-1} \int_{0}^{t}\|x-y\|_{C[0, s]} d s \leqslant$ $\|x-y\|_{C[0, t]}$ доказательство теоремы 3 сохраняется вплоть до вывода соотношения (5.13), которое здесь переписывается в виде

$$
\begin{aligned}
& L F_{K, 1}(t)-L G(t) \\
& \qquad \int_{0}^{t} \frac{\alpha b}{s} \int_{0}^{s} \| \Psi\left(F_{K, 1}(\cdot)\right)-\Psi(G(\cdot)) \\
& \quad-\int_{0}^{\cdot}\left(\Psi_{v}\left(F_{K, 1}(\cdot-v)\right) \Psi_{v}(G(\cdot-v))\right) \Phi(d v) \|_{C[0, v]} d v d s \\
& \leqslant \alpha \beta b(1+\Phi(t)) \int_{0}^{t} \frac{1}{s} \int_{0}^{s}\left\|F_{K, 1}-G\right\|_{C[0, v]} d v d s .
\end{aligned}
$$


Следовательно, при $t \in[0, T-u]$

$$
\begin{aligned}
& \mathbf{E}\left\|F_{K, 1}-G\right\|_{C[0, t]} \\
& \quad \leqslant \alpha S(K) t+\alpha \beta b(1+\Phi(T-u)) \int_{0}^{t} \frac{1}{s} \int_{0}^{s} \mathbf{E}\left\|F_{K, 1}-G\right\|_{C[0, v]} d v d s
\end{aligned}
$$

Докажем вспомогательное утверждение: пусть гладкая функция у удовлетворяет неравенству $\left(t y^{\prime}\right)^{\prime}(t) \leqslant p t+q y(t)$ при некоторых $p, q>0$ для любого $t$ из $\left[0, T^{\prime}\right]$ и краевым условиям $y(0)=0, y^{\prime}(0)=0$, тогда $y^{\prime}(t) \leqslant y_{\max }^{\prime}(t)$ и $y(t) \leqslant y_{\max }(t)$ при $t \in\left[0, T^{\prime}\right]$, где $y_{\max }(t)-$ peшение дифференциального уравнения $\left(t y_{\max }^{\prime}\right)^{\prime}(t)=p t+q y_{\max }(t)$ с краевыми условиями $y_{\max }(0)=0, y_{\max }^{\prime}(0)=0$. Действительно, положив $f(t)=y^{\prime}(t)-y_{\max }^{\prime}(t)$, имеем неравенство $(t f(t))^{\prime} \leqslant q \int_{0}^{t} f(s) d s$, интегрируя которое, приходим к соотношениям

$$
f(t) \leqslant \frac{q}{t} \int_{0}^{t} \int_{0}^{s} f(v) d v d s=q \int_{0}^{t} f(v) \frac{t-v}{t} d v \leqslant q \int_{0}^{t} f(v) d v .
$$

Используя лемму 3 , получаем, что $f(t) \leqslant 0$, т.е. $y^{\prime}(t) \leqslant y_{\max }^{\prime}(t)$, так что в силу краевых условий $y(t) \leqslant y_{\max }(t)$. Осталось найти $y_{\max }$. Будем искать $y_{\max }$ в виде ряда $y_{\max }(t)=\sum_{n=2}^{+\infty} a_{n} t^{n}$. Подставляя этот ряд в дифференциальное уравнение, приходим к тождеству

$$
\sum_{n=2}^{+\infty} n(n-1) a_{n} t^{n-1}+\sum_{n=2}^{+\infty} n a_{n} t^{n-1}=p t+q \sum_{n=2}^{+\infty} a_{n} t^{n} .
$$

Приравнивая коэффициенты при одинаковых степенях $t$, получаем $n^{2} a_{n}=q a_{n-1}$ при $n>2$ и $4 a_{2}=p$, и, следовательно, $y_{\max }(t)=$ $p q^{-2} \sum_{n=2}^{+\infty}(q t)^{n} /(n !)^{2}=p q^{-2} g_{1}(q t)$.

Возвращаясь к доказательству теоремы, положим

$$
y(t)=\int_{0}^{t} s^{-1} \int_{0}^{s} \mathbf{E}\left\|F_{K, 1}-G\right\|_{C[0, v]} d v d s
$$

тогда $\left(t y^{\prime}(t)\right)^{\prime}=\mathbf{E}\left\|F_{K, 1}-G\right\|_{C[0, t]}$. Заметим также, что из непрерывности функции $\mathbf{E}\left\|F_{K, 1}-G\right\|_{C[0, t]}$ и равенства $\mathbf{E}\left\|F_{K, 1}-G\right\|_{C[0,0]}=0$ следуют краевые условия $y(0)==0, y^{\prime}(0)=0$. Воспользуемся доказанным утверждением. В данном случае, согласно $(5.18), q=\alpha \beta b(1+\Phi(T-u))=g_{0}(T-u)$, a $p=S(K)$, поэтому неравенство $y \leqslant y_{\max }$ влечет

$$
\mathbf{E}\left\|F_{K, 1}-G\right\|_{C[0, t]} \leqslant \alpha S(K) t+\frac{\alpha S(K) g_{1}\left(g_{0}(T-u) t\right)}{g_{0}(T-u)} .
$$

С учетом неравенств

$$
\int_{0}^{t} \mathbf{E}\left\|F_{K, 1}-G\right\|_{C[0, s]} d s \leqslant t y^{\prime}(t) \leqslant t y_{\max }^{\prime}(t)=\alpha S(K) t \frac{g_{1}^{\prime}\left(g_{0}(T-u) t\right)}{g_{0}(T-u)}
$$


из $(5.4)-(5.12),(5.17),(5.18)$ имеем

$$
\begin{aligned}
\left|\mathbf{E} H\left(\xi_{K}^{T}\right)-\mathbf{E} H\left(\mu^{T}\right)\right| & \\
\leqslant \frac{\sqrt{a_{K}}+\sqrt{b_{K}}}{\sqrt{K}}+\alpha \beta b_{K} S(K) \int_{0}^{T} & \left(T-u+\frac{g_{1}\left(g_{0}(T-u)(T-u)\right)}{g_{0}(T-u)}\right. \\
& \left.+(T-u) \frac{g_{1}^{\prime}\left(g_{0}(T-u)(T-u)\right)}{g_{0}(T-u)}\right) \Phi(d u) .
\end{aligned}
$$

Последнее неравенство завершает доказательство теоремы.

3 а м е ч а н и е 6 . Доказательства теорем 3 и 4 используют идеи [7], но имеются и существенные отличия. А именно, вместо неравенства для $\left\|L_{K, 1} h-L h\right\|_{C[0, t]}$ работы [7] мы применяем леммы 1 и 2 , поскольку для оценки $\left\|\mathscr{Z}_{K, 1} h-\mathscr{Z} h\right\|_{C[0, s]}$ нужен анализ более сложного объекта, введенного в лемме 2. Отметим также, что неравенства в теоремах 3 и 4 представляют интерес, когда их правые части малы, т.е. при $T$ порядка $\ln K$ и $g_{1}^{-1}\left(K^{\frac{1}{3}}\right)$ соответственно.

Автор особенно благодарен своему научному руководителю А. В. Булинскому за постановку задачи, ценные замечания и советы, а также очень признателен А. П. Шашкину за плодотворное обсуждение работы.

\section{СПИСОК ЛИТЕРАТУРЫ}

1. Bartlett M. S. Some evolutionary stochastic processes. - J. Roy. Statist. Soc., Ser. B, 1949, v. 11, p. 211-229.

2. Barbour A.D. On a functional central limit theorem for Markov population processes. - Adv. Appl. Probab., 1974, v. 6, p. 21-39.

3. Sellke T. On the asymptotic distribution of the size of a stochastic epidemic. - J. Appl. Probab., 1983, v. 20, № 2, p. 390-394.

4. Becker N. The uses of epidemic models. - Biometrics, 1979, v. 35, p. 295-305.

5. Mollison D. Epidemic Models: Their Structure and Relation to Data. Cambridge: Cambridge Univ. Press, 1995.

6. Reinert G. The asymptotic evolution of the General Stochastic Epidemic. - Ann. Appl. Probab., 1995, v. 5, № 4, p. 1061-1086.

7. Reinert G. Stein's method for epidemic processes. - Complex Stochastic Systems. Ed. by O. E. Barndorff-Nielsen, D. R. Cox, and C. Klüppelberg. Boca Raton: Chapman \& Hall/CRC, 2001, p. 235-275.

8. Reinert $G$. A weak law of large numbers for empirical measures via Stein's method. Ann. Probab., 1995, v. 23, № 1, p. 334-354.

9. Massart P. The tight constant in the Dvoretzky-Kiefer-Wolfowitz inequality. - Ann. Probab., 1990, v. 18, № 3, p. 1269-1283.

10. Абрамович М., Стиган И. Справочник по специальным функциям с формулами, графиками и математическими таблицами. М.: Наука, 1979, 830 с.

11. Dudley R. M. Uniform Central Limit Theorems. Cambridge: Cambridge Univ. Press, 1999, $436 \mathrm{c}$.

12. Vapnik V.N. Statistical Learning Theory. New York: Wiley, 1998, 736 p. 